COMUNICAÇÃOCIENTÍFICA

\title{
ATIVIDADE RESPIRATÓRIA E PRODUÇÃO DE ETILENO EM LARANJA 'PÊRA' SUBMETIDA A NÍVEIS DE PROCESSAMENTO MÍNIMO E TEMPERATURAS DE ARMAZENAMENTO ${ }^{1}$
}

\author{
MARIACECÍLIADEARRUDA ${ }^{2}$, ANGELO PEDRO JACOMINO $^{3}$, ANALUIZA PINHEIRO $^{4}$, \\ MARCOS JOSÉ TREVISAN ${ }^{5}$, EDWIN MOISÉS MARCOS ORTEGA ${ }^{6}$
}

RESUMO-O objetivo deste trabalho foi avaliar o efeito de diferentes níveis de processamento e temperaturas de armazenamento na atividade respiratória e na produção de etileno de laranja 'Pêra' minimamente processada. O experimento foi realizado em duas etapas. Na primeira, as laranjas lavadas, sanificadas e resfriadas foram submetidas aos processamentos: a) segmentos; b) inteiras sem albedo; c) inteiras com albedo; d) intactas (controle). As laranjas minimamente processadas foram armazenadas a $6^{\circ} \mathrm{C}$. Na segunda etapa, as laranjas sem albedo foram armazenadas a $1 ; 11 ; 21$ e $31^{\circ} \mathrm{C}$. A atividade respiratória e a produção de etileno eram determinadas imediatamente após o processamento; a cada hora, durante 10 horas, e a cada 24 horas, durante sete dias. O delineamento utilizado foi o inteiramente casualizado, com seis repetições por tratamento. O processamento interferiu na atividade respiratória, que foi maior imediatamente após o descascamento e a separação dos segmentos. O processamento das laranjas na forma inteira, com ou sem albedo, afetou a atividade respiratória das mesmas somente nas primeiras horas após o processamento. Durante todo período de armazenamento, a atividade respiratória das laranjas a $1^{\circ} \mathrm{e} 11^{\circ} \mathrm{C}$ não diferiu entre si, sendo inferior à das laranjas a $21^{\circ} \mathrm{e} 31^{\circ} \mathrm{C}$. O etileno foi detectado apenas nos frutos mantidos a $21^{\circ} \mathrm{e} 31^{\circ} \mathrm{C}$. Os quocientes de temperatura, após a estabilização, eram 1,73 para $1-11^{\circ} \mathrm{C}, 2,11$ para $11-21^{\circ} \mathrm{C}$, e 1,54 para $21-31^{\circ} \mathrm{C}$. A atividade respiratória das laranjas foi influenciada pelos níveis de processamento e pela temperatura de armazenamento.

Termos para indexação: Citrus sinensis; Respiração; Processamento mínimo.

\section{RESPIRATORY ACTIVITY AND ETHYLENE PRODUCTION IN 'PERA'ORANGE SUBMITTED TO DIFFERENT LEVELS OF PROCESSING AND STORAGE TEMPERATURE}

\begin{abstract}
The present work was aimed at evaluating the effect of different processing levels and storage temperatures on the respiratory activity and the ethylene production of minimally processed 'Pera' orange. The study was carried out in two stages. The first consisted of submitting the oranges previously washed, sanitized and chilled to the following processing: a) segments; b) whole without albedo; c) whole with albedo; d) intact (control). The minimally processed oranges were stored at $6^{\circ} \mathrm{C}$. In the second stage oranges without albedo were stored at $1,11,21$ and $31^{\circ} \mathrm{C}$. The respiratory activity and ethylene production were determined immediately after the closure of the jars, at every hour for 10 hours and at every 24 hours for 7 days. The statistical design was completely randomized with six replicates per treatment. Processing influenced the respiratory activity at greater rates immediately after peeling and segments separation. The processing of oranges, in form with or without albedo affected the respiratory activity only during the first hours after processing. During all storage period, the respiratory activities of oranges at $1^{\circ}$ and $11^{\circ} \mathrm{C}$ did not differ between them, and were lower than oranges at $21^{\circ}$ and $31^{\circ} \mathrm{C}$. Ethylene was only detected in fruits stored at $21^{\circ}$ and $31^{\circ} \mathrm{C}$. The temperature quotients after stabilization were 1.73 for $1-11^{\circ} \mathrm{C} ; 2.11$ for $11-21^{\circ} \mathrm{C}$ and 1.54 for $21-31^{\circ} \mathrm{C}$. The respiratory activity of oranges was influenced by the processing levels and by the storage temperature.
\end{abstract}

Index Terms: Citrus sinensis, Respiration, Minimal processing.

Frutas e hortaliças minimamente processadas mantêm seus tecidos vivos e não exibem a mesma resposta fisiológica que um tecido inteiro (Wiley, 1994). Os danos físicos causados aos tecidos pelas operações de processamento resultam em aumento no metabolismo dos vegetais, com conseqüente aumento na atividade respiratória e na produção de etileno (Rosen $\&$ Kader, 1989). Tendo-se que, quanto mais intenso o metabolismo do produto, maior será sua perecibilidade, sendo o controle desse processo crucial para obtenção de produtos com qualidade.

Uma das primeiras respostas dos frutos ao estresse do processamento é o aumento na produção de etileno, acompanhado de aumento na atividade respiratória e modificações, geralmente indesejáveis, na aparência, sabor, aroma

${ }^{1}$ (Trabalho 013-08). Recebido em: 07-01-2008. Aceito para publicação em 29-07-2008.

${ }^{2}$ Engenheira agrônoma, Doutora em Agronomia, APTA Pólo Centro Oeste, Av. Rodrigues Alves, 40-40, 17030-000 - Bauru-SP - mcarruda@apta.sp.gov.br; ${ }^{3}$ Engenheiro agrônomo, Doutor em Agronomia, Professor da ESALQ/USP, Av. Pádua Dias, 11, C.P 09, Piracicaba-SP - jacomino@esalq.usp.br; ${ }^{4}$ Cientista de Alimentos, Mestre em Fisiologia e Bioquímica de Plantas, ESALQ/USP - alpinhei@esalq.usp.br;

${ }^{5}$ Engenheiro agrônomo, Mestre em Agronomia, ESALQ/USP - mjtrevis@esalq.usp.br;

${ }^{6}$ Estatístico, Doutor em Estatística, Professor da ESALQ/USP - edwin@esalq.usp.br 
e textura (Wiley, 1994). Vários trabalhos, como os de Watada et al. (1996), Sarzi et al. (2001), Jacomino et al. (2002) e Moreira (2005), evidenciam que o aumento no metabolismo do produto é tanto maior quanto maior a temperatura de armazenamento e/ou quanto maior o nível de processamento.

As mudanças bioquímicas em frutas e hortaliças minimamente processadas são, em parte, conseqüência do efeito da temperatura na atividade enzimática. Em temperaturas maiores que $10^{\circ} \mathrm{C}$, a produção de $\mathrm{CO}_{2}$ aumenta abruptamente devido à intensificação do metabolismo e proliferação microbiana. Baixas temperaturas durante o processamento e armazenamento retardam o metabolismo do vegetal, com diminuição de sua atividade respiratória e enzimática (Wiley, 1994).

Quanto menor a temperatura de armazenamento, melhor será a conservação dos produtos minimamente processados, sem problemas de injúrias pelo frio, visto que esses produtos apresentam vida útil curta e são mantidos sob refrigeração até o consumo.

A laranja é a fruta mais produzida no Brasil e apresenta potencial para ser minimamente processada. Embora seja um fruto não-climatérico, com baixo metabolismo, há necessidade de conhecer seu comportamento fisiológico quando minimamente processada e armazenada. O objetivo deste trabalho foi determinar a atividade respiratória e a produção de etileno em laranjas 'Pêra' minimamente processadas em função do nível de processamento e da temperatura de armazenamento.

Este trabalho foi conduzido em duas etapas. Em ambas as etapas, o processamento foi realizado em sala climatizada $\left(18^{\circ} \mathrm{C}\right)$, adotando-se as boas práticas de fabricação, com higienização dos utensílios, do ambiente e utilização de toucas, aventais, máscaras, luvas e botas por parte dos operadores. $\mathrm{Na}$ etapa 1, as laranjas, provenientes de pomares comerciais da região de Engenheiro Coelho, foram lavadas, sanificadas (200 mg. $\mathrm{L}^{-1} \mathrm{de}$ cloro por 10 minutos), resfriadas a $6^{\circ} \mathrm{C}$ por 12 horas e submetidas aos processamentos: a) segmentos; b) inteiras sem albedo; c) inteiras com albedo; d) inteiras com flavedo (controle). Esses produtos foram acondicionados em jarros que permitem fechamento hermético e armazenados em B.O.D a $6^{\circ} \mathrm{C}$. Na etapa 2 , as laranjas foram processadas inteiras sem albedo, acondicionadas em jarros e armazenadas em B.O.D. a 1; 11; $21 \mathrm{e}$ $31^{\circ} \mathrm{C}$.

Para a obtenção de laranjas inteiras com albedo, foi realizado o descascamento mecânico, o que consistiu em fixar a laranja num pequeno equipamento que permite girar a fruta, enquanto uma lâmina retira o flavedo na forma de uma tira contínua. Na obtenção das laranjas inteiras sem albedo, o descascamento foi realizado utilizando-se de tratamento hidrotérmico (imersão das laranjas resfriadas a $6^{\circ} \mathrm{C}$ em água a $50^{\circ} \mathrm{C}$, por 8 minutos), com posterior descasque manual. Os segmentos foram obtidos de laranjas inteiras e sem albedo, utilizando-se de espátula de inox para auxiliar na separação dos segmentos.

O delineamento utilizado foi o inteiramente casualizado, com seis repetições por tratamento. Cada repetição foi representada por um jarro contendo um fruto de aproximadamente $200 \mathrm{~g}$.
Determinaram-se a atividade respiratória e a produção de etileno imediatamente após o processamento; a cada hora, durante um período de 10 horas; e posteriormente a cada 24 horas, por sete dias. Os jarros foram fechados durante 30 minutos antes de cada coleta, e no restante do tempo, permaneceram abertos. Amostras de $1 \mathrm{~mL}$ de gás foram retiradas do interior dos jarros, através de um septo de silicone com seringa modelo Gastight, marca Hamilton de 2,5ml, e injetadas em cromatógrafo a gás Thermoffinigan, modelo Trace GC 2000, equipado com coluna Porapack $\mathrm{N} \mathrm{a} 100^{\circ} \mathrm{C}$, sendo de $4 \mathrm{~m}$ de comprimento para detecção de $\mathrm{CO}_{2}$, com hidrogênio como gás de arraste a um fluxo de 39,1 mL.min ${ }^{-1}$ e 1,8 m para $\mathrm{C}_{2} \mathrm{H}_{4}$, com nitrogênio a $33,3 \mathrm{~mL} \cdot \mathrm{min}^{-1}, 2$ injetores a $100^{\circ} \mathrm{C}$, metanador para o $\mathrm{CO}_{2}$ a $350^{\circ} \mathrm{C}$ e 2 detectores de ionização de chama (FID a $250^{\circ} \mathrm{C}$ ). $\mathrm{O}$ aparelho foi calibrado, usando-se padrões contendo 2150 e $29900 \mu \mathrm{L} \mathrm{CO}_{2} . \mathrm{L}^{-1}$ e $1.94 \mu \mathrm{L}$ $\mathrm{C}_{2} \mathrm{H}_{4} \cdot \mathrm{L}^{-1}$.

Os resultados expressos em $\mu$ L.L.-1 de $\mathrm{CO}_{2}$ e de $\mathrm{C}_{2} \mathrm{H}_{4}$ foram utilizados para o cálculo da atividade respiratória e da produção de etileno, levando-se em consideração o volume do jarro, a massa do fruto e o tempo que os jarros permaneceram fechados. O quociente de temperatura foi determinado com base na atividade respiratória dos produtos, utilizando a seguinte fórmula: $\mathrm{Q}_{10}=$ $(\mathrm{R} 2 / \mathrm{R} 1)^{10 /(\mathrm{T} 2-\mathrm{T} 1)}$, onde: $\mathrm{R} 1\left(\mathrm{~T}^{\circ} \mathrm{C}\right)=$ atividade respiratória à temperatura $\mathrm{T}^{\circ} \mathrm{C}$ e $\mathrm{R} 2\left(\mathrm{~T}+10^{\circ} \mathrm{C}\right)=$ atividade respiratória à temperatura $\mathrm{T}+10^{\circ} \mathrm{C}$.

Os resultados foram submetidos à análise de variância, e as médias entre tratamentos foram comparadas pelo teste de Tukey (5\%).

Na etapa 1, observou-se que os segmentos de laranja apresentaram maior respiração $(\mathrm{P}<0,05)$ que as laranjas intactas e as submetidas aos outros níveis de processamento, durante todo o período de armazenamento (Figura 1). Imediatamente após o processamento, houve elevação de 8,3 vezes na atividade respiratória dos segmentos de laranja em relação às laranjas intactas. Para as laranjas com e sem albedo, esse aumento foi de 2,8 e 2,3 vezes, respectivamente, em relação às laranjas intactas. A elevação na respiração está relacionada ao estresse físico causado por ocasião do processamento (Rosen \& Kader, 1989).

A maior atividade respiratória observada nos segmentos, logo após o processamento e ao longo do armazenamento, devese ao maior estresse sofrido, pois, para a obtenção dos segmentos, os frutos, além de descascados, foram submetidos à operação de separação dos segmentos, que danificou parte da membrana que reveste as vesículas de suco.

A maior atividade respiratória nas laranjas com albedo que nas sem albedo foi observada somente no momento do processamento (Figura 1) e não foi significativa $(\mathrm{P}>0,05)$.

A respiração dos segmentos de laranja tendeu a estabilizar-se na quarta hora após o processamento, com valores médios de $7 \mathrm{mLCO}{ }_{2} \mathrm{Kg}^{-1} \cdot \mathrm{h}^{-1}$. A estabilização da respiração nas laranjas com e sem albedo ocorreu na terceira hora após o processamento, quando a atividade respiratória assumiu valores semelhantes ao do fruto intacto, ou seja, o estresse perdurou por pouco tempo (Figura 1A). Donadon (2005) verificou que laranjas descascadas manual, mecânica ou enzimaticamente apresentaram elevação na atividade respiratória na segunda hora 
após o processamento, seguida de redução e estabilização. A atividade respiratória de tangores 'Murcott' descascados igualou-se à dos frutos com casca, cinco horas após o processamento (Moreira, 2005).

Não detectou-se etileno nas laranjas submetidas aos diferentes níveis de processamento.

$\mathrm{Na}$ etapa 2 , logo após o processamento, a atividade respiratória dos produtos foi de 11,$6 ; 15,8 ; 27,6$ e $48,3 \mathrm{~mL}$ $\mathrm{CO}_{2} \cdot \mathrm{kg}^{-1} \cdot \mathrm{h}^{-1}$, respectivamente a $1 ; 11 ; 21 \mathrm{e} 31^{\circ} \mathrm{C}$ (Figura $2 \mathrm{~A}$ ).

Durante todo o período de armazenamento, a atividade respiratória das laranjas sem albedo a $1^{\circ}$ e $11^{\circ} \mathrm{C}$ não diferiu entre si $(\mathrm{P}>0,05)$, sendo inferior àquelas a $21^{\circ}$ e $31^{\circ} \mathrm{C}(\mathrm{P}<0,05)$.

A alta atividade respiratória das laranjas sem albedo a $31^{\circ} \mathrm{C}$ reduziu-se drasticamente até a quarta hora, quando, então, tendeu a se estabilizar. Os produtos a $11^{\circ}$ e a $21^{\circ} \mathrm{C}$ apresentaram redução até a segunda hora, e produtos a $1^{\circ} \mathrm{C}$, redução na primeira hora (Figura 2A). Sarzi et al. (2001), que trabalharam com mamão em fatias e em metades, armazenado a $3 ; 6$ e $9^{\circ} \mathrm{C}$, verificaram que, na primeira hora após o corte, os produtos apresentaram pico na respiração, que se reduziu na hora seguinte e estabilizou-se.

No quarto e no sexto dias de armazenamento, foi detectado início de crescimento de fungos filamentosos, Penicillium e Cladosporium, nas laranjas a $31^{\circ} \mathrm{C}$ e $21^{\circ}$, respectivamente. Concomitantemente ao aparecimento de fungos, observou-se elevação na atividade respiratória das laranjas a $21^{\circ} \mathrm{C}$ (Figura 2B). Watada et al. (1996) observaram aumento drástico na respiração de melões dos grupos reticulatus e inodorus, minimamente processados, mantidos a $20^{\circ} \mathrm{C}$. Esses autores atribuíram a alta atividade respiratória à deteriorações fisiológicas e crescimento microbiano.

O etileno foi detectado apenas nos frutos mantidos a $21^{\circ}$ e $31^{\circ} \mathrm{C}$, mas com detecção inconstante ao longo do período de armazenamento e com picos cromatográficos indefinidos. Os valores encontrados foram, em média,de $0,39 \mathrm{uL} . \mathrm{L}^{-1}$ para laranjas a $21^{\circ} \mathrm{C}$, e $0,76 \mathrm{uL} \cdot \mathrm{L}^{-1}$ para as mantidas a $31^{\circ} \mathrm{C}$.

Os quocientes de temperatura determinados após a estabilização foram de 1,73 para a faixa $1^{\circ}-11^{\circ} \mathrm{C} ; 2,11$ para a de $11^{\circ}$ $21^{\circ} \mathrm{C}$, e 1,54 para a de $21^{\circ}-31^{\circ} \mathrm{C}$. Watada et al. (1996) também observaram maior valor de $\mathrm{Q}_{10}$ na faixa de 10 a $20^{\circ} \mathrm{C}$, do que na de 0 a $10^{\circ} \mathrm{C}$, em 11 produtos minimamente processados, de um total de 15 produtos estudados. Esses autores afirmam que o maior valor de $\mathrm{Q}_{10}$ na faixa de 10 a $20^{\circ} \mathrm{C}$ se deve à rápida deterioração dos produtos a $20^{\circ} \mathrm{C}$. Assim, destaca-se a importância da manutenção de baixas temperaturas durante o manuseio e armazenamento de produtos minimamente processados.

Moreira (2005), que trabalhou com tangores 'Murcott' descascados, também relatou maiores valores de quocientes de temperatura para a faixa de $11-21^{\circ} \mathrm{C}$ do que nas demais faixas de temperatura estudadas.

Para a maioria dos produtos, o $\mathrm{Q}_{10}$ encontra-se entre 2,0 e 2,5 , com variação de temperatura de $5^{\circ}$ a $25^{\circ} \mathrm{C}$. No entanto, os valores de $\mathrm{Q}_{10}$ podem sofrer variações com o aumento da temperatura, sendo que o aumento de $25^{\circ} \mathrm{C}$ para $30^{\circ}$ ou $35^{\circ} \mathrm{C}$ promove redução no valor de $\mathrm{Q}_{10}$ para a maioria dos produtos, possivelmente em decorrência da redução da atividade enzimática (Chitarra \& Chitarra, 2005).

O processamento mínimo de laranjas nas formas inteiras, com ou sem albedo, afeta a atividade respiratória somente nas primeiras horas após o processamento, enquanto os segmentos apresentam maior metabolismo e, provavelmente, menor vida útil. Além disso, sua obtenção é dificultada, gerando grande perda de produto.
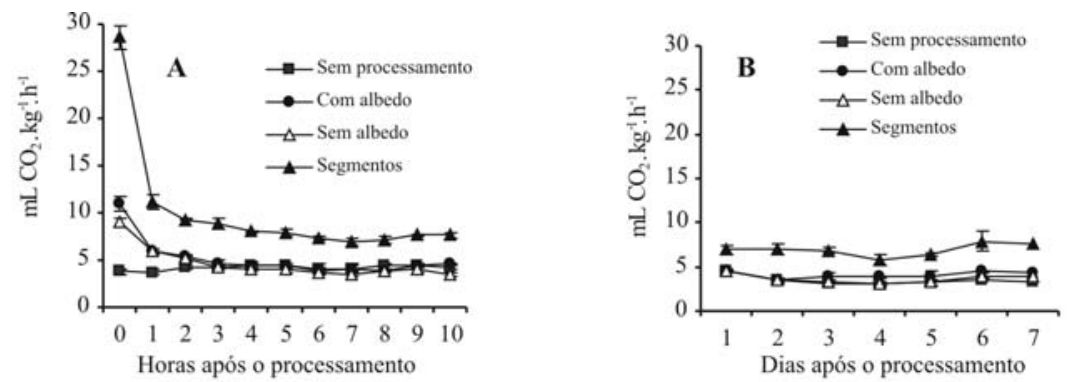

FIGURA 1 - Atividade respiratória de laranja 'Pêra' minimamente processada, armazenada a $6^{\circ} \mathrm{C}$. As barras representam o desviopadrão da média.
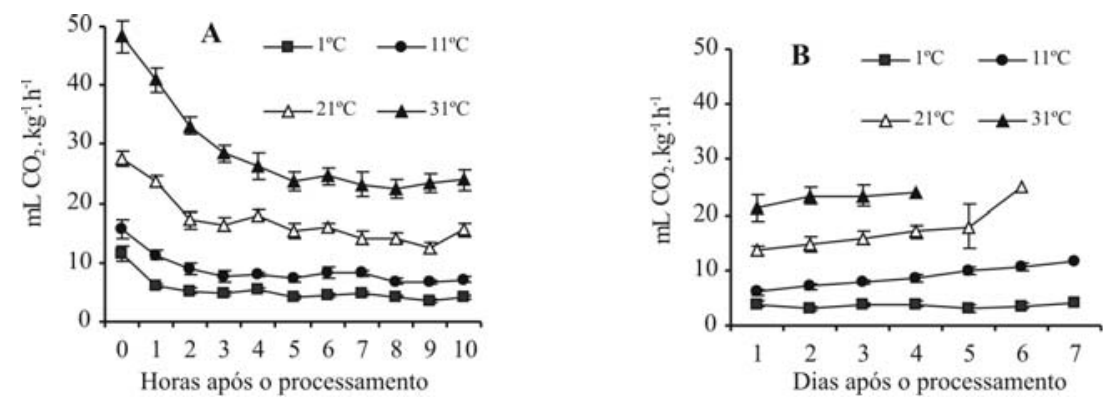

FIGURA 2 - Atividade respiratória de laranja 'Pêra' minimamente processada e armazenada a $1 ; 11 ; 21$ e $31^{\circ} \mathrm{C}$. As barras representam o desvio-padrão da média. 


\section{REFERÊNCIAS}

CHITARRA, M.I.F.; CHITARRA, A.B. Pós-colheita de frutos e hortaliças: fisiologia e manuseio. 2. ed. Lavras: UFLA, 2005. 785p.

DONADON, J.R. Conservação de frutas cítricas minimamente processadas: tipos de descasque e temperaturas de armazenamento. 2005. 76 f. Dissertação (Mestrado em Produção Vegetal) - Universidade Estadual Paulista, Faculdade de Ciências Agronômicas e Veterinária, Jaboticabal, 2005.

JACOMINO, A.P.; ARRUDA, M.C.de; CARON, V.C.; KLUGE, R.A. Taxa respiratória de melão inteiro e minimamente processado armazenado em três temperaturas. Horticultura Brasileira, Brasília, v.20, n.2, p.402, 2002. Suplemento 1.

MOREIRA, R.C. Processamento mínimo de tangor 'Murcott': caracterização fisiológica e recobrimentos comestíveis. 2005. 85 f. Dissertação (Mestrado em Fitotecnia) - Escola Superior de Agricultura 'Luiz de Queiroz', Universidade de São Paulo, Piracicaba, 2005.
ROSEN, J.; KADER, A.A. Postharvest physiology and quality maintenance of slice pear and strawberry fruits. Journal of Food Science, Chicago, v.54, p.656-659, 1989.

SARZI, B.; DURIGAN, J.F.; LIMA, M.A.; MATTIUZ, B. Comportamento respiratório de mamão minimamente processado quando armazenado sob diferentes temperaturas. In: CONGRESSOBRASILEIRODEFISIOLOGIA VEGETAL, 8., 2001, Ilhéus. Anais...Ilhéus: Sociedade Brasileira de Fisiologia, 2001. CD-ROM.

WATADA, A.E.; KO, N.P.; MINOTT, D.A. Factors affecting quality of fresh-cut horticultural products. Postharvest Biology and Technology, Amsterdam, v.9, n.2, p.115-125, 1996.

WILEY, R.C. Minimally processed refrigerated fruits and vegetables. New York: Chapman \& Hall, 1994. 368p. 